MISE AU POINT

\title{
Sécurisation du circuit des médicaments utilisés pour les chimiothérapies
}

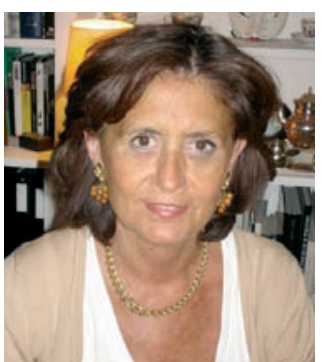

-Martine Poivre et Anne-Sophie Glouzmann

Pharmaciens, Clinique Lambert,

92250 La Garenne Colombes

Les médicaments utilisés dans les protocoles de chimiothérapie sont des médicaments dangereux, il faut les manipuler avec précautions, en suivant les recommandations des Bonnes Pratiques de préparation des médicaments (2007), en répondant aux exigences de la HAS (V2010) et de l'arrêté du 6 avril 2011 relatif au management de la prise en charge médicamenteuse et aux médicaments dans les établissements de santé.

es médicaments antinéoplasiques et cytotoxiques, Let certains anticorps monoclonaux sont des médicaments dangereux, c'est-à-dire qu'en raison de leur toxicité propre, ils présentent un danger pour le personnel mais aussi pour le patient et l'environnement. La liste de ces médicaments dangereux doit être affichée dans les services responsables de la préparation et de l'administration de ces médicaments; les directives concernant la sécurité de la manipulation et la protection contre les agents cancérigènes doivent être appliquées.

Ces préparations doivent être réalisées de façon aseptique en évitant tout contact du produit avec l'environnement lors de son transfert.

- Les sources et voies d'exposition à ces médicaments sont multiples et présentes tout au long du circuit du médicament dans l'établissement de soin, de sa livraison à la pharmacie à son administration.

Des particules de médicaments peuvent être présentes sur les emballages et flacons " mal lavés », ce qui peut contaminer l'environnement dès la réception des produits à la pharmacie. Maintenant les industriels doivent garantir que les surfaces externes des flacons sont exemptes de contamination, utiliser des containers de transport évitant toute possibilité de casse de flacon, et prendre toutes les mesures pour éviter toute contamination en cas de bris de flacon. Le pharmacien doit privilégier l'industriel le plus vigilant à ces recommandations.

Les toxicités engendrées par ces produits sont soit immédiates, avec des réactions locales (rougeurs, nécroses) ou générales (prurit avec rash érythémateux ou réactions urticantes des membres supérieurs, œedème palpébral, ulcérations de la muqueuse nasale, réactions asthmatiformes, vertiges...), soit retardées (avortements spontanés...).

- Il faut donc sécuriser le circuit de ces médicaments dangereux.

\begin{tabular}{|c|c|c|}
\hline Sources & Voies & Où et quand \\
\hline Particules présentes sur les emballages de flacons de médicaments & cutanée & Pharmacie au déballage \\
\hline Particules présentes sur les flacons de médicaments & cutanée & Unité de reconstitution \\
\hline Flacons cassés & cutanée & $\begin{array}{l}\text { Pharmacie au déballage } \\
\text { et Unité de reconstitution }\end{array}$ \\
\hline Aérosols dus à l'augmentation de pression dans le flacon de médicament & \multirow{3}{*}{$\begin{array}{l}\text { inhalation de } \\
\text { gouttelettes, particules, } \\
\text { vapeurs; yeux, } \\
\text { muqueuses }\end{array}$} & Unité de reconstitution \\
\hline Aérosols dus à la purge de l'air d'une seringue & & Unité de reconstitution \\
\hline $\begin{array}{l}\text { Fuite au niveau de la tubulure, de la seringue ; fuites au niveau des } \\
\text { septums de flacons à la suite de ponctions répétées... }\end{array}$ & & Unité de reconstitution \\
\hline Fuite au niveau de la tubulure, de la seringue... & cutanée & Administration au patient \\
\hline Déchets souillés & cutanée & Élimination des déchets \\
\hline
\end{tabular}

L'arrêté du 6 avril 2011 précise que la « prise en charge médicamenteuse est un processus combinant des étapes pluridisciplinaires et interdépendantes visant I'utilisation sécurisée, appropriée et efficiente du médicament chez le patient » et chaque étape, de la prescription à l'administration en passant par l'approvisionnement, la préparation, le circuit du médicament doit être sécurisé et une attention particulière est portée sur les médicaments à risque et les patients à risque. Le pharmacien, garant du circuit du médicament, doit tout mettre en œuvre pour limiter les risques de contamination chimique, l'établissement doit mettre en place les techniques nécessaires pour pouvoir appliquer des procédures sûres de manipulation des médicaments dangereux.

Plusieurs fournisseurs proposent des dispositifs spéciaux améliorant la sécurisation de la préparation de ces médicaments dangereux.

- Les étapes à sécuriser :

- Réception et stockage des médicaments dangereux à la pharmacie : les livraisons de ces médicaments sont identifiés par des étiquettes spéciales sur le carton permettant ainsi aux réceptionnistes de prendre les précautions nécessaires lors du déballage et du rangement des produits en stock: mettre des gants spécifiques, manipuler avec précaution, ranger dans un espace réservé aux médicaments dangereux ; un kit en « cas de bris de flacon ou écrasement de comprimé » doit être à leur disposition immédiate et doit contenir tout le matériel nécessaire pour ramasser un flacon cassé avec le maximum de sécurité

- Transport des produits en interne : il est réalisé par des personnes averties, dans des conditions ne présentant aucun risque pour les personnes et l'environnement et dans des conditions maintenant la qualité du produit (température, protection contre la lumière...), dans des containers spécifiques, fermés et identifiables.

- Préparation du médicament : les personnes de I'unité de reconstitution doivent revêtir une tenue protectrice adaptée, en fonction du type (hotte à flux laminaire vertical ou isolateur). Lors de la reconstitution, les préparateurs doivent manipuler en respectant les procédures pour éviter les projections et aérosols dans l'environnement ; il existe à présent de nombreux dispositifs améliorant la sécurité à chaque étape de la préparation et permettant de ne plus utiliser d'aiguille : les dispositifs de prélèvement sur le conditionnement primaire, les adaptateurs de seringues, système permettant le prélèvement et le transfert d'un produit stérile dans un autre contenant stérile..

- Les préparations sont sécurisées avec les systèmes clos. Il y a obligation de mettre à l'entrée des salles de reconstitution un logo spécifique indiquant que des produits dangereux sont manipulés.

- Délivrance au service de soin : dans un sachet protecteur étiqueté au nom du patient, on met le médicament préparé prêt à l'emploi, c'est-à-dire avec le dispositif d'administration connecté et purgé avec le solvant de dilution afin que le personnel infirmier puisse réaliser l'administration sans risque. Les préparations sont transportées par des personnes averties, dans des conditions ne présentant aucun risque pour les personnes et l'environnement et dans des conditions maintenant la qualité du produit (température, délai, protection contre la lumière...), dans des containers spécifiques, fermés et identifiables.

- Administration du médicament au patient : les infirmiers doivent revêtir une tenue adaptée protectrice pour la connexion et la déconnexion des chimiothérapies ; il existe à présent des dispositifs améliorant la sécurité de l'administration, il s'agit d'un système « arbre » : perfuseur par gravité adapté à la poche de rinçage et sur lequel I'IDE branche directement le médicament préparé et délivré, prêt à l'emploi, par l'unité de reconstitution ; ainsi lors de la connexion et de la déconnexion, tout contact de I'IDE avec le médicament à risque toxique est limité. À la fin de l'administration du médicament, le système doit être retiré non démonté.

Élimination dans les déchets en respectant les procédures mises en place dans l'établissement ; élimination des excréta, revêtir une tenue protectrice appropriée. Dans le cadre des contaminations microbiologique, chimique et l'exposition professionnelle, les nombreux dispositifs sécurisés mis sur le marché améliorent la sécurisation des préparations et de l'administration des médicaments dangereux. En fonction des besoins et des objectifs recherchés, avant toute décision, il faut mettre en place une étude et évaluer le surcoût engendré par ces nouveaux dispositifs. • 